\title{
Insuficiente evidencia para recomendar tratamiento de tres días a pacientes con neumonía aguda no severa de la comunidad
}

\section{Objetivo}

Evaluar la eficacia de tres días de tratamiento antibiótico endovenoso comparado con ocho días, en adultos internados con neumonía adquirida de la comunidad (NAC) leve a moderada.

\section{Diseño}

Ensayo clínico aleatorizado, controlado y doble ciego.

Lugar

Nueve hospitales de los Países Bajos, entre 2000 y 2003.

\section{Participantes}

Se incluyeron 186 pacientes mayores de 18 años con signos clínicos y radiológicos de NAC, temperatura mayor a $38^{\circ} \mathrm{C}$ e índice de severidad de 110 o menor o mayores de 65 años con clínica positiva, anormalidades radiológicas e índice de severidad menor o igual a 110. Ver tabla 1.

Se excluyeron embarazadas, pacientes con alergia a amoxicilina, inmunodeprimidos o esperanza de vida menor a mes mes, tratamiento antibiótico las 24 horas previas a la consulta, co-infecciones, ingreso hospitalario reciente, insuficiencia respiratoria, requerimiento de cuidados intensivos, empiema, sospecha de broncoaspiración, y neumonía atípica por Klebsiella o Staphylococo.

Tabla 1: Regla de predicción de severidad y pronóstico a 30 días en pacientes con neumonía aguda de la comunidad'

\begin{tabular}{|c|c|}
\hline Garacterística pronostica por grupo & Puntaje* \\
\hline $\begin{array}{l}\text { - Factores Demográficos } \\
\text { Edad Hombres } \\
\text { Mujeres edad (años) } \\
\text { Residente en hogar tercera edad }\end{array}$ & $\begin{array}{l}\text { Edad en años } \\
\text { Edad en años } \\
-10+10\end{array}$ \\
\hline $\begin{array}{l}\text { - Comorbilidades } \\
\text { Enfermedad neoplásica } \\
\text { Enfermedad hepática } \\
\text { Insuficiencia cardíca } \\
\text { Enfermedad cerebrovascular } \\
\text { Enfermedad renal }\end{array}$ & $\begin{array}{l}+30 \\
+20 \\
+10 \\
+10 \\
+10\end{array}$ \\
\hline $\begin{array}{l}\text { - Exámen físico } \\
\text { Estado mental alterado } \\
\text { Frecuencia respiratoria mayor o igual a 30/min } \\
\text { Presion arterial sistólica menor a 90mmHg } \\
\text { Temperatura menor a } 35 \mathrm{C} \text { o mayor a } 40 \mathrm{C} \\
\text { Pulso mayor a } 125 / \mathrm{min}\end{array}$ & $\begin{array}{l}+20 \\
+20 \\
+15 \\
+15 \\
+10 \\
\end{array}$ \\
\hline 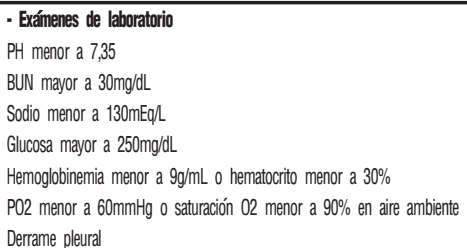 & $\begin{array}{l}+30 \\
+20 \\
+20 \\
+10 \\
+10 \\
+10 \\
+10\end{array}$ \\
\hline
\end{tabular}

${ }^{*}$ Riesgo de muerte a los 30 días según el puntaje. Clase I (menos de 51 ): 0,1 a 0,4\%; Clase II (51 a 70): 0,6 a 0,7\%; Clase III (71 a 90): 0,9 a 2,8\%; Clase IV (91 a 130): 9,3 a 12,5\%; Clase V (más de 130): 27 a 31\%

\section{Intervención}

A todos los pacientes se les realizó tratamiento empírico con amoxicilina endovenosa durante 72 horas, tras lo cual se evaluó la mejoría de síntomas respiratorios (disnea, tos, expectoración y color del esputo) y el estado general. Si mejoraban y toleraban la vía oral eran aleatorizados a recibir amoxicilina $(n=63) 750 \mathrm{mg}$ vía oral cada 8 h por cinco días o placebo $(n=56)$.

Se evaluó a los pacientes al inicio y a los 3,7,10,14 y 28 días.

\section{Medición de resultados}

El resultado primario fue la tasa de éxito terapéutico al día 10 (mejoría clínica y ausencia de necesidad de tratamiento antibiótico posterior). El estudio fue diseñado para evaluar la no inferioridad del tratamiento corto en relación al de ocho días, definido como una diferencia en la tasa de mejoría clínica menor al $10 \%$ entre los grupos.

El análisis se realizó por intención de tratar y por protocolo. Como resultados secundarios se midieron las tasas de éxito terapéutico al día 28 , la severidad de los síntomas, las tasas de éxito radiológicas y bacteriológicas a los diez y 28 días, la permanencia en el hospital y los eventos adversos.

\section{Resultados}

Las características basales de ambos grupos eran comparables, salvo en la severidad de los síntomas: el grupo intervención presentó mayor severidad al inicio del estudio. Ambos grupos presentaron tasas de curación similares a los diez días (ver tabla 2).

La resolución de los síntomas fue similar entre los grupos, así como la tasa de curación radiológica a los diez días (diferencia 3\%: IC95\%: 10 a 16) y a los 28 días (diferencia 6\%, IC95\%; -7\% a 20\%). Seis pacientes (11\%) en el grupo placebo y $13(21 \%)$ en el grupo intervención presentaron eventos adversos $(p=0,1)$.

Tabla 2: tasas de curación en ambos grupos

\begin{tabular}{|c|c|c|c|c|}
\hline \multirow{3}{*}{ DIA 10} & Tasa de curación & Placebo & Amoxicilina & Diferencia (IC $95 \%$ ) \\
\hline & Por protocolo & $50,54(93 \%)$ & $56160 \quad(93 \%)$ & $0.1 \quad(-9$ a 10$)$ \\
\hline & Por intención de tratar & $5056(89 \%)$ & $5663(89 \%)$ & $0.4 \quad(-11$ a 12$)$ \\
\hline \multirow{2}{*}{ DA 28} & Por protocolo & $4752(90 \%)$ & $49 / 56 \quad(88 \%)$ & $2(-9 a-15)$ \\
\hline & Por intención de tratar & $47,56 \quad(84 \%)$ & $49 / 63(78 \%)$ & $6(-8$ a 20) \\
\hline
\end{tabular}

\section{Conclusiones}

El tratamiento corto de tres días con amoxicilina endovenosa no es inferior al de ocho días para el tratamiento de pacientes hospitalizados por NAC leve a moderada.

Fuente de financiamiento: Glaxo SmithKline fue el proveedor de la amoxicilina. Los autores recibieron un subsidio del Healthcare insurance board, Amstelveen, Netherlands. 
Los consensos recientes para el tratamiento de la NAC están apoyados por evidencia de buena calidad ${ }^{2,3}$, sin embargo el tema de la duración del tratamiento es materia de debate. La recomendación actual es tratar durante siete a diez días la neumonía no complicada, y en el caso de iniciar antibioticoterapia parenteral, pasar a la vía oral cuando los síntomas hayan mejorado, desaparezca la fiebre y se haya reducido la leucocitosis, siempre y cuando la absorción gastrointestinal sea normal ${ }^{2}$. Sin embargo, existen ventajas en reducir la duración del tratamiento, como la disminución de las tasas de resistencia antibiótica, la menor tasa de eventos adversos y el menor costo del tratamiento.

Si bien los resultados de este trabajo son promisorios en este sentido, debemos ser cautelosos en la interpretación de estos hallazgos, ya que los autores no describen el cálculo de poder estimado, punto clave en estudios que intentan demostrar equivalencia o no inferioridad. En este caso, al reportar que no existe diferencia entre los grupos no podemos saber si el hallazgo refleja la realidad o habría sido necesario incluir más pacientes para detectarla. Los autores definen como clínicamente relevante una diferencia del
$10 \%$ entre los tratamientos. Para estimarla con un poder de al menos $80 \%$, deberían haber incluido 280 pacientes por grupo.

Coincidiendo con eso, observamos que la amplitud de los intervalos de confianza presentados sobrepasan el límite del $10 \%$ que los autores fijaron.

Otro punto que puede generar debate es la hospitalización y la prescripción de antibióticos por vía parenteral a pacientes con NAC leve, que habitualmente son tratados por vía oral y en forma ambulatoria, punto que va en contra de la aplicabilidad de los resultados a la práctica diaria.

\section{Conclusión de las comentadoras}

Es necesario obtener información más precisa mediante un ensayo clínico con más cantidad de pacientes antes de recomendar el tratamiento de tres días con amoxicilina endovenosa en pacientes hospitalizados por NAC leve a moderada.

\section{Marcela López y Victoria Wurcel. [ Unidad de Medicina Familiar y Preventiva del Hospital Italiano de Buenos Aires. ]}

López M. y Wurcel V. Insuficiente evidencia para recomendar tratamiento de tres días a pacientes con neumonía aguda no severa de la comunidad. Evid. actual. pract. ambul. 9(4);103-104. Jul-Ag 2006. Comentado de Moussaoui, R et al. Effectiveness of discontinuing antibiotic treatment after three days versus eight days in mild to moderate-severe community acquired pneumonia: randomised, double blind study. BMJ 2006;332:1355. PMID: 16763247.

\section{Referencias}

1. Fine MJ, Auble TE, Yealy DM, Hanusa BH, Weissfeld LA, Singer DE, et al. A prediction rule to identify low-risk patients with community-acquired pneumonia. N Engl J Med 1997; 336.

2. Luna C. y col. Neumonía adquirida en la comunidad en adultos, guía de práctica clínica para la Argentina. Revista MEDICINA (Buenos Aires) 1997; 57: 343355.

3. BTS guidelines for the management of community acquired pneumonia in adults. Thorax 2001;56(suppl 4):IV 1-64.

\section{INFO - EVIDENCIA}

\section{¿Qué es Evidencia, Actualización en la Práctica Ambulatoria?}

Una publicación independiente editada cada dos meses desde 1997 por la Fundación MF, Organización sin fines de lucro dedicada a promover el desarrollo de la medicina familiar y la atención primaria de la salud.

\section{¿Cuál es el objetivo de Evidencia?}

Contribuir a la educación continua y a la actualización de los profesionales de la salud de la región en el área de la atención ambulatoria.

\section{¿Contenidos de Evidencia}

- Información independiente, actualizada y resumida en forma sencilla y en castellano sobre temas relevantes de la atención ambulatoria.

Artículos seleccionados por su calidad y relevancia clínica, resumidos y comentados críticamente por destacados profesionales del área.

- Revisiones sobre temas clínicos, epidemiológicos, sanitarios o humanísticos, de importancia para la práctica ambulatoria.

Notas farmacológicas para la actualización rápida de temas de terapéutica clínica.

- Discusión de casos clínicos con herramientas de Medicina Basada en la Evidencia.

Glosario de términos de epidemiología y medicina basada en la evidencia

\section{¿Cómo es posible acceder a Evidencia?}

Por suscripción a la edición en papel, o bien por Intenet, en nuestro sitio:

http://www.evidencia.org 